October 31, 2013

\title{
On the Diagonal Susceptibility of the 2D Ising Model
}

\author{
Craig A. Tracy \\ Department of Mathematics \\ University of California \\ Davis, CA 95616, USA \\ Harold Widom \\ Department of Mathematics \\ University of California \\ Santa Cruz, CA 95064, USA
}

\begin{abstract}
We consider the diagonal susceptibility of the isotropic 2D Ising model for temperatures below the critical temperature. For a parameter $k$ related to temperature and the interaction constant, we extend the diagonal susceptibility to complex $k$ inside the unit disc, and prove the conjecture that the unit circle is a natural boundary.
\end{abstract}

\section{Introduction}

For the 2D Ising model [15, 16, 23], after the zero-field free energy [20] and the spontaneous magnetization [21, 28], the most important zero-field thermodynamic quantity is the magnetic susceptibility $\chi$. Since the free energy is known only in zero magnetic field, the susceptibility is usually studied through its relation with the zero-field spin-spin correlation function:

$$
\beta^{-1} \chi=\sum_{M, N \in \mathbb{Z}}\left\{\left\langle\sigma_{0,0} \sigma_{M, N}\right\rangle-\mathcal{M}^{2}\right\}
$$

where $\beta=\left(k_{B} T\right)^{-1}, T$ is temperature, $k_{B}$ is Boltzmann's constant and $\mathcal{M}$ is the spontaneous magnetization. If $T_{c}$ denotes the critical temperature, we recall that for the isotropic 2D Ising model, i.e. horizontal and vertical interaction constants have the same value $J$, the spontaneous magnetization is given for $T<T_{c}$ by

$$
\mathcal{M}=\left(1-k^{2}\right)^{1 / 8}
$$

where $k:=(\sinh 2 \beta J)^{-2}$ and $\mathcal{M}$ is zero for $T>T_{c}$. (For $0<T<T_{c}$ we have $0<k<1$.) 
The analysis of $\chi=\chi(T)$ in the neighborhood of the critical temperature $T_{c}$ has a long history. We refer the reader to McCoy et al. [17] for a review of these developments. The analysis of $\chi$ for complex temperatures was initiated by Guttmann and Enting [13] and by Nickel [18, 19]. (For further developments see [10, 22].) Nickel's analysis takes as its beginning the (commonly called) form-factor or particle expansion of the spin-spin correlation function [27]. For $T<T_{c}$ this expansion is an infinite sum whose $n$th summand is a $2 n$-dimensional integral. From an asymptotic analysis of these integrals, Nickel was led to conjecture that $|k|=1$ is a natural boundary for $\chi$. As Nickel himself noted, the analysis is nonrigorous since one must show that there are no cancellations of singularities in the sum. This has turned out to be a difficult problem to resolve rigorously.

In Boukraa et al. [7], these authors, building on results of [14, introduce a simplified model for $\chi$, called the diagonal susceptibility $\chi_{d}$, which is defined by having "a magnetic field which acts only on one diagonal of the lattice." (See 2] for further developments.) Thus the analogue of (1) is

$$
\beta^{-1} \chi_{d}=\sum_{N \in \mathbb{Z}}\left\{\left\langle\sigma_{0,0} \sigma_{N, N}\right\rangle-\mathcal{M}^{2}\right\} .
$$

In this paper we consider $\chi_{d}$ only for $T<T_{c}$, in which case $k<1$. Then we extend $\chi_{d}$ to $k$ complex with $|k|<1$. Using the Toeplitz determinant representation of the diagonal correlations [15, 24], we first derive the known representation of $\chi_{d}$ in terms of a sum of multiple integrals $\mathcal{S}_{n}$. The derivation is different from those in [8, 9], [14] and [26]. As in [26] we use the identity of Geronimo-Case [12] and Borodin-Okounkov [5] relating a Toeplitz determinant to the Fredholm determinant of a product of Hankel operators. (For simplified proofs of the GCBO formula, see [3, 6].) But here we go from there to the multiple integral representation directly using a general identity for the integral of a product of determinants [1] (see eqn. (1.3) in [25]). For further background on the relationship between Toeplitz determinants and Ising correlations, see [4, 11].

In Section 4 we show that for each root of unity $\epsilon \neq \pm 1$ a certain derivative of a certain $\mathcal{S}_{n}$ is unbounded as $k \rightarrow \epsilon$ radially, while the same derivative of the sum of the other $\mathcal{S}_{n}$ remains bounded (Lemma 4 ). Thus, the unit circle $|k|=1$ is a natural boundary for $\chi_{d}$. This proves the conjecture by Boukraa et al. [7]. We note that in [7] the authors present an argument that the singularity of $\mathcal{S}_{n}$ at an $n$th root of unity $\epsilon$ is of the form $(k-\epsilon)^{2 n^{2}-1} \log (k-\epsilon)$. Lemma 2 in Section IV formalizes this statement and fills in details of the proof. 


\section{Toeplitz determinant representation}

It was shown in [15, 24] that for $N>1$ the diagonal correlation has a representation as an $N \times N$ Toeplitz determinant:

$$
\left\langle\sigma_{0,0} \sigma_{N, N}\right\rangle=\operatorname{det}\left(\varphi_{m-n}\right)_{1 \leq m, n \leq N} .
$$

Here

$$
\varphi(\xi)=\left[\frac{1-k \xi^{-1}}{1-k \xi}\right]^{1 / 2}
$$

and

$$
\varphi_{m}=\frac{1}{2 \pi i} \int \varphi(\xi) \xi^{-m-1} d \xi
$$

with integration over the unit circle. (We have $\left\langle\sigma_{0,0}^{2}\right\rangle=1$.)

As in [26] we invoke the formula of Geronimo-Case [12] and Borodin-Okounkov [5] to write the Toeplitz determinant in terms of the Fredholm determinant of a product of Hankel operators. We have $\varphi(\xi)=\varphi_{+}(\xi) \varphi_{-}(\xi)$, where

$$
\varphi_{+}(\xi)=(1-k \xi)^{-1 / 2} \text { and } \varphi_{-}(\xi)=\left(1-k \xi^{-1}\right)^{1 / 2}
$$

Since $|k|<1$ these extend analytically inside and outside the unit circle, respectively. The square roots are determined by $\varphi_{+}(0)=\varphi_{-}(\infty)=1$.

The Hankel operator $H(\psi)$ is the operator on $\ell^{2}\left(\mathbb{Z}^{+}\right)$with kernel $\left(\psi_{i+j+1}\right)_{i, j \geq 0}$, where $\psi_{m}$ given in analogy with (44). The operator $H_{N}(\psi)$ has kernel $\left(\psi_{N+i+j+1}\right)$.

Using $\varphi_{ \pm}(\xi)=1 / \varphi_{\mp}\left(\xi^{-1}\right)$, we find that the formula of GCBO gives

$$
\operatorname{det}\left(\varphi_{m-n}\right)_{1 \leq m, n \leq N}=\mathcal{M}^{2} \operatorname{det}\left(I-H_{N}\left(\frac{\varphi_{-}}{\varphi_{+}}\right) H_{N}\left(\frac{\varphi_{+}}{\varphi_{-}}\right)\right) \text {. }
$$

Thus, if we define

$$
\Lambda(\xi)=\frac{\varphi_{-}(\xi)}{\varphi_{+}(\xi)}=\sqrt{(1-k \xi)(1-k / \xi)}, \quad K_{N}=H_{N}(\Lambda) H_{N}\left(\Lambda^{-1}\right)
$$

then

$$
\beta^{-1} \chi_{d}=1-\mathcal{M}^{2}+2 \mathcal{M}^{2} \sum_{N=1}^{\infty}\left[\operatorname{det}\left(I-K_{N}\right)-1\right]=1+\mathcal{M}^{2}(2 \mathcal{S}-1)
$$

where

$$
\mathcal{S}=\sum_{N=1}^{\infty}\left[\operatorname{det}\left(I-K_{N}\right)-1\right]
$$

In what follows we extend $\Lambda$ to be holomorphic in the complex plane cut along $[0, k] \cup\left[k^{-1}, \infty\right]$. 


\section{Formula for $\mathcal{S}$}

We use a slightly different notation for Hankel operators here.

Proposition. Let $H_{N}(d u)$ and $H_{N}(d v)$ be two Hankel matrices acting on $\ell^{2}\left(\mathbb{Z}^{+}\right)$ with $i, j$ entries

$$
\int x^{N+i+j} d u(x), \quad \int y^{N+i+j} d v(y)
$$

respectively, where $u$ and $v$ are measures supported inside the unit circle. Set $K_{N}=$ $H_{N}(d u) H_{N}(d v)$. Then

$$
\begin{gathered}
\sum_{N=1}^{\infty}\left[\operatorname{det}\left(I-K_{N}\right)-1\right] \\
=\sum_{n=1}^{\infty} \frac{(-1)^{n}}{(n !)^{2}} \int \cdots \int \frac{\prod_{i} x_{i} y_{i}}{1-\prod_{i} x_{i} y_{i}}\left(\operatorname{det}\left(\frac{1}{1-x_{i} y_{j}}\right)\right)^{2} \prod_{i} d u\left(x_{i}\right) d v\left(y_{i}\right),
\end{gathered}
$$

where indices in the integrand run from 1 to $n$.

Proof. The Fredholm expansion is

$$
\operatorname{det}\left(I-K_{N}\right)=1+\sum_{n=1}^{\infty} \frac{(-1)^{n}}{n !} \sum_{p_{1}, \ldots, p_{n} \geq 0} \operatorname{det}\left(K_{N}\left(p_{i}, p_{j}\right)\right)
$$

Therefore its suffices to show that

$$
\begin{gathered}
\sum_{N=1}^{\infty} \sum_{p_{1}, \ldots, p_{n} \geq 0} \operatorname{det}\left(K_{N}\left(p_{i}, p_{j}\right)\right) \\
=\frac{1}{n !} \int \cdots \int \frac{\prod_{i} x_{i} y_{i}}{1-\prod_{i} x_{i} y_{i}}\left(\operatorname{det}\left(\frac{1}{1-x_{i} y_{j}}\right)\right)^{2} d u\left(x_{1}\right) \cdots d u\left(x_{n}\right) d v\left(y_{1}\right) \cdots d v\left(y_{n}\right) .
\end{gathered}
$$

We have

$$
K_{N}\left(p_{i}, p_{j}\right)=\iint \frac{x^{N+p_{i}} y^{N+p_{j}}}{1-x y} d u(x) d v(y) .
$$

It follows by a general identity [1] (eqn. (1.3) in [25]) that

$$
\begin{gathered}
\operatorname{det}\left(K_{N}\left(p_{i}, p_{j}\right)\right)=\frac{1}{n !} \int \cdots \int \operatorname{det}\left(x_{i}^{N+p_{j}}\right) \operatorname{det}\left(y_{i}^{N+p_{j}}\right) \prod_{i} \frac{1}{1-x_{i} y_{i}} \prod_{i} d u\left(x_{i}\right) d v\left(y_{i}\right) \\
=\frac{1}{n !} \int \cdots \int\left(\prod_{i} x_{i} y_{i}\right)^{N} \operatorname{det}\left(x_{i}^{p_{j}}\right) \operatorname{det}\left(y_{i}^{p_{j}}\right) \prod_{i} \frac{1}{1-x_{i} y_{i}} \prod_{i} d u\left(x_{i}\right) d v\left(y_{i}\right) .
\end{gathered}
$$

Summing over $N$ gives 


$$
\begin{gathered}
\sum_{N=1}^{\infty} \operatorname{det}\left(K_{N}\left(p_{i}, p_{j}\right)\right)= \\
\frac{1}{n !} \int \cdots \int \frac{\prod_{i} x_{i} y_{i}}{1-\prod_{i} x_{i} y_{i}} \operatorname{det}\left(x_{i}^{p_{j}}\right) \operatorname{det}\left(y_{i}^{p_{j}}\right) \prod_{i} \frac{1}{1-x_{i} y_{i}} \prod_{i} d u\left(x_{i}\right) d v\left(y_{i}\right) .
\end{gathered}
$$

(Interchanging the sum with the integral is justified since the supports of $u$ and $v$ are inside the unit circle.)

Now we sum over $p_{1}, \ldots, p_{n} \geq 0$. Using the general identity again (but in the other direction) gives

$$
\sum_{p_{1}, \ldots, p_{n} \geq 0} \operatorname{det}\left(x_{i}^{p_{j}}\right) \operatorname{det}\left(y_{i}^{p_{j}}\right)=n ! \operatorname{det}\left(\sum_{p \geq 0} x_{i}^{p} y_{j}^{p}\right)=n ! \operatorname{det}\left(\frac{1}{1-x_{i} y_{j}}\right) .
$$

We almost obtained the desired result. It remain to show that

$$
\operatorname{det}\left(\frac{1}{1-x_{i} y_{j}}\right) \prod_{i} \frac{1}{1-x_{i} y_{i}}
$$

which we obtain in the integrand, may be replaced by

$$
\frac{1}{n !}\left(\operatorname{det}\left(\frac{1}{1-x_{i} y_{j}}\right)\right)^{2}
$$

This follows by symmetrization over the $x_{i}$. (The rest of the integrand is symmetric.) For a permutation $\pi$, replacing the $x_{i}$ by $x_{\pi(i)}$ multiplies the determinant in (8) by $\operatorname{sgn} \pi$, so to symmetrize we replace the other factor by

$$
\frac{1}{n !} \sum_{\pi} \operatorname{sgn} \pi \frac{1}{1-x_{\pi(i)} y_{i}}=\frac{1}{n !} \operatorname{det}\left(\frac{1}{1-x_{i} y_{j}}\right) .
$$

Thus, symmetrizing (8) gives (9).

We apply this to the operator $K_{N}=H_{N}(\Lambda) H_{N}\left(\Lambda^{-1}\right)$ given by (5). The matrix for $H_{N}(\Lambda)$ has $i, j$ entry

$$
\frac{1}{2 \pi i} \int \Lambda(\xi) \xi^{-N-i-j-2} d \xi
$$

where the integration may be taken over a circle with radius in $\left(1,|k|^{-1}\right)$. Setting $\xi=1 / x$ and using $\Lambda(1 / x)=\Lambda(x)$ we see that the entries of $H_{N}(\Lambda)$ are given as in (7) with

$$
d u(x)=\frac{1}{2 \pi i} \Lambda(x) d x
$$


and integration is over a circle $\mathcal{C}$ with radius in $(|k|, 1)$. Similarly $H_{N}\left(\Lambda^{-1}\right)=H_{N}(v)$ where in (7)

$$
d \psi(y)=\frac{1}{2 \pi i} \Lambda(y)^{-1} d y
$$

with integration over the same circle $\mathcal{C}$.

Hence the Proposition gives

$$
\mathcal{S}=\sum_{n=1}^{\infty} \mathcal{S}_{n}
$$

where

$$
\mathcal{S}_{n}=\frac{(-1)^{n}}{(n !)^{2}} \frac{1}{(2 \pi i)^{2 n}} \int \cdots \int \frac{\prod_{i} x_{i} y_{i}}{1-\prod_{i} x_{i} y_{i}}\left(\operatorname{det}\left(\frac{1}{1-x_{i} y_{j}}\right)\right)^{2} \prod_{i} \frac{\Lambda\left(x_{i}\right)}{\Lambda\left(y_{i}\right)} \prod_{i} d x_{i} d y_{i}
$$

with all integrations over $\mathcal{C}$.

We deform $\mathcal{C}$ to the contour back and forth along the interval $[0, k]$, and then make the substitutions $x_{i} \rightarrow k x_{i}, y_{i} \rightarrow k y_{i}$. We obtain

$\mathcal{S}_{n}=\frac{1}{(n !)^{2}} \frac{\kappa^{2 n}}{\pi^{2 n}} \int_{0}^{1} \cdots \int_{0}^{1} \frac{\prod_{i} x_{i} y_{i}}{1-\kappa^{n} \prod_{i} x_{i} y_{i}}\left(\operatorname{det}\left(\frac{1}{1-\kappa x_{i} y_{j}}\right)\right)^{2} \prod_{i} \frac{\Lambda_{1}\left(x_{i}\right)}{\Lambda_{1}\left(y_{i}\right)} \prod_{i} d x_{i} d y_{i}$,

where we have set

$$
\kappa=k^{2}, \quad \Lambda_{1}(x)=\sqrt{\frac{(1-x)(1-\kappa x)}{x}} .
$$

Using the fact that the determinant in the integrand is a Cauchy determinant we obtain the alternative expression

$$
\mathcal{S}_{n}=\frac{1}{(n !)^{2}} \frac{\kappa^{n(n+1)}}{\pi^{2 n}} \int_{0}^{1} \cdots \int_{0}^{1} \frac{\prod_{i} x_{i} y_{i}}{1-\kappa^{n} \prod_{i} x_{i} y_{i}} \frac{\Delta(x)^{2} \Delta(y)^{2}}{\prod_{i, j}\left(1-\kappa x_{i} y_{j}\right)^{2}} \prod_{i} \frac{\Lambda_{1}\left(x_{i}\right)}{\Lambda_{1}\left(y_{i}\right)} \prod_{i} d x_{i} d y_{i}
$$

where $\Delta(x)$ and $\Delta(y)$ are Vandermonde determinants. Clearly, $\mathcal{S}_{n}$ is holomorphic in $\kappa$ for $|\kappa|<1$. It is straightforward to prove that the sum (10) converges uniformly in $\kappa$ for $|\kappa| \leq r$ for all $0<r<1$; and hence, $\mathcal{S}$ is holomorphic in the $\kappa$ unit disc.

\section{Natural boundary}

Theorem. The unit circle $|\kappa|=1$ is a natural boundary for $\mathcal{S}$.

There will four lemmas. In these, $\epsilon \neq 1$ will be an $n$th root of unity and we consider the behavior of $\mathcal{S}$ as $\kappa \rightarrow \epsilon$ radially. 
For $\ell \geq 0$ we use the representation (12) and look at

$$
\int_{0}^{1} \cdots \int_{0}^{1} \frac{\prod_{i} x_{i} y_{i}}{\left(1-\kappa^{n} \prod_{i} x_{i} y_{i}\right)^{\ell+1}} \frac{\Delta(x)^{2} \Delta(y)^{2}}{\prod_{i, j}\left(1-\kappa x_{i} y_{j}\right)^{2}} \prod_{i} \frac{\Lambda_{1}\left(x_{i}\right)}{\Lambda_{1}\left(y_{i}\right)} \prod_{i} d x_{i} d y_{i},
$$

where all indices run from 1 to $n$. This will be the main contribution to $d^{\ell} \mathcal{S}_{n} / d \kappa^{\ell}$.

Lemma 1. The integral (13) is bounded when $\ell<2 n^{2}-1$ and it is of the order $\log (1-|\kappa|)^{-1}$ when $\ell=2 n^{2}-1$.

Proof. First we establish the first part of the statement. The numerator in the first factor is bounded and the denominator in the second factor is bounded away from zero as $\kappa \rightarrow \epsilon$ since $\epsilon \neq 1$.

If $\prod_{i} x_{i} y_{i}<1-\delta$ then the rest of the integrand is bounded except for the last quotient, and the integral of that is $O(1)$.

If $\prod_{i} x_{i} y_{i}>1-\delta$ then each $x_{i}, y_{i}>1-\delta$ and the integrand has absolute value at most a constant times

$$
\frac{\Delta(x)^{2} \Delta(y)^{2}}{\left|\kappa^{-n}-\prod_{i} x_{i} y_{i}\right|^{\ell+1}} \prod_{i} \sqrt{\frac{1-x_{i}}{1-y_{i}}}
$$

We assumed that $\kappa \rightarrow \epsilon$ along a radius, so $\kappa^{-n}>1$. Therefore we get an upper bound if we replace $\kappa^{-n}$ by 1 . Then in the integral we make the substitutions $x_{i}=$ $1-\xi_{i}, y_{i}=1-\eta_{i}\left(\right.$ so $\left.\xi_{i}, \eta_{i}<\delta\right)$ and we obtain

$$
\frac{\Delta(\xi)^{2} \Delta(\eta)^{2}}{\left(1-\prod_{i}\left(1-\xi_{i}\right)\left(1-\eta_{i}\right)\right)^{\ell+1}} \prod_{i} \sqrt{\frac{\xi_{i}}{\eta_{i}}}
$$

Whenever $z_{i} \in[0,1](i=1, \ldots, m)$ we have $z_{1} \cdots z_{m} \leq z_{i}$ for each $i$, and so averaging gives

$$
z_{1} \cdots z_{m} \leq\left(\sum_{j} z_{j}\right) / m
$$

and therefore

$$
1-z_{1} \cdots z_{m} \geq \sum_{j}\left(1-z_{j}\right) / m
$$

It follows that

$$
1-\prod_{i}\left(1-\xi_{i}\right)\left(1-\eta_{i}\right) \geq \sum_{i}\left(\xi_{i}+\eta_{i}\right) / 2 n
$$

Therefore the integrand above is at most $(2 n)^{\ell+1}$ times

$$
\frac{\Delta(\xi)^{2} \Delta(\eta)^{2}}{\left(\sum_{i}\left(\xi_{i}+\eta_{i}\right)\right)^{\ell+1}} \prod_{i} \sqrt{\frac{\xi_{i}}{\eta_{i}}}
$$


This is homogeneous of degree $2 n(n-1)-\ell-1$. We first integrate over the region $\sum_{i}\left(\xi_{i}+\eta_{i}\right)=r$ and then over $r$. The resulting integral is at most a constant times

$$
\int_{0}^{2 n \delta} r^{2 n^{2}-\ell-2} d r
$$

This is finite when $\ell<2 n^{2}-1$, and so the first statement of the lemma is established. We note that the $(2 n-1)$-dimensional volume of the region $\sum_{i}\left(\xi_{i}+\eta_{i}\right)=1$ is $1 / \Gamma(2 n)$, another nice factor which we can use if needed. But it won't be.

We now consider the integral when $\ell=2 n^{2}-1$. As before, the integral over the region $\prod_{i} x_{i} y_{i}<1-\delta$ is $O(1)$, so we assume $\prod_{i} x_{i} y_{i}>1-\delta$. In particular each $x_{i}, y_{i}>1-\delta$. The factors $1-\kappa x_{i} y_{j}$ in the second denominator equal $1-\kappa(1+O(\delta))=$ $(1-\kappa)(1+O(\delta))$ since $\kappa$ is bounded away from 1 . From this we see that if we factor out $\kappa^{2 n^{3}}$ from the first denominator and $(1-\kappa)^{n^{2}}$ from the second, the integrand becomes

$$
\frac{\Delta(x)^{2} \Delta(y)^{2}}{\left(\kappa^{-n}-\prod_{i} x_{i} y_{i}\right)^{2 n^{2}}} \prod_{i} \sqrt{\frac{1-x_{i}}{1-y_{i}}}(1+O(\delta)) .
$$

We again make the substitutions $x_{i}=1-\xi_{i}, y_{i}=1-\eta_{i}$ and set $r=\sum_{i}\left(\xi_{i}+y_{i}\right)$. Then since $\prod_{i}\left(1-\xi_{i}\right)\left(1-\eta_{i}\right)=1-r+O\left(r^{2}\right)$ the integrand becomes

$$
\frac{\Delta(\xi)^{2} \Delta(\eta)^{2}}{\left(\kappa^{-n}-1+r+O\left(r^{2}\right)\right)^{2 n^{2}}} \prod_{i} \sqrt{\frac{\xi_{i}}{\eta_{i}}}(1+O(\delta)) .
$$

The integration domain $\prod_{i} x_{i} y_{i}>1-\delta$ becomes $r+O\left(r^{2}\right)<\delta$, which is contained in $r<2 \delta$ and contains $r<\delta / 2$. The integral without the $O(\delta)$ term is at least a constant times

$$
\int_{0}^{\delta / 2} \frac{r^{2 n^{2}-1}}{\left(\kappa^{-n}-1+2 r\right)^{2 n^{2}}} d r
$$

which is asymptotically a constant independent of $\delta$ times $\log \left(\kappa^{-n}-1\right)^{-1}$ as $\kappa \rightarrow \epsilon$. Similarly the integral of the $O(\delta)$ term is at most a constant independent of $\delta$ times $\delta \log \left(\kappa^{-n}-1\right)^{-1}$. Since $\delta$ is arbitrarily small, this proves the lemma.

Lemma 2. We have

$$
\left(\frac{d}{d \kappa}\right)^{2 n^{2}-1} \mathcal{S}_{n} \approx \log (1-|\kappa|)^{-1} .
$$

Proof. To compute the derivative of the integral in (12) one integral we get is a constant depending on $n$ times (13) with $\ell=2 n^{2}-1$. The other integrals are similar but in each the power in the denominator is less than $2 n^{2}-1$ while we get extra factors obtained by differentiating the rest of the integrand for $\mathcal{S}_{n}$. These factors are of the form $\left(1-\kappa x_{i} y_{i}\right)^{-1},\left(1-\kappa x_{i}\right)^{-1}$, or $\left(1-\kappa y_{i}\right)^{-1}$. By an obvious modification 
of the first statement of Lemma 1 we see that these other integrals are all bounded. The lemma follows.

Lemma 3. If $\epsilon^{m} \neq 1$ then

$$
\left(\frac{d}{d \kappa}\right)^{2 n^{2}-1} \mathcal{S}_{m}=O(1)
$$

Proof. If $\epsilon^{m} \neq 1$ all integrands obtained by differentiating the integral in (12) are bounded as $\kappa \rightarrow \epsilon$.

Lemma 4. We have

$$
\sum_{m>n}\left(\frac{d}{d \kappa}\right)^{2 n^{2}-1} S_{m}=O(1)
$$

Proof. We shall show that for $\kappa$ sufficiently close to $\epsilon$ all integrals we get by differentiating the integral for $S_{m}$ are at most $A^{m} m^{m}$, where $A$ is some constant. Note that the value of $A$ will change with each of its appearances. In may depend on $n$, but not on $m$. Because of the $1 /(m !)^{2}$ appearing in front of the integrals this will show that the sum is bounded.

As before, we first use (12) (with $n$ replaced by $m$ ) and consider the integral we get when the first factor in the integrand is differentiated $2 n^{2}-1$ times. All indices in the integrands now run from 1 to $m$.

First,

$$
\left|1-\kappa^{m} \prod_{i} x_{i} y_{i}\right|=\left|\kappa^{m}\right|\left|\kappa^{-m}-\prod_{i} x_{i} y_{i}\right| \geq|\kappa|^{m}\left(1-\prod_{i} x_{i} y_{i}\right)
$$

Next, $\left|1-\kappa x_{i}\right| \leq 2$. Since $y_{i} \in[0,1]$ and $\kappa \in[0, \epsilon]$ we also have $\kappa y_{i} \in[0, \epsilon]$. Therefore $\left|1-\kappa y_{i}\right| \geq a$, where $a=\operatorname{dist}(1,[0, \epsilon])$. Hence the integrand in (12) after differentiating the first factor has absolute value at most $A^{m}$ times

$$
\frac{1}{\left(1-\prod x_{i} y_{i}\right)^{2 n^{2}}} \frac{\Delta(x)^{2} \Delta(y)^{2}}{\prod_{i, j}\left|1-\kappa x_{i} y_{j}\right|^{2}} \prod_{i} \sqrt{\frac{1-x_{i}}{1-y_{i}} \frac{y_{i}}{x_{i}}} .
$$

Since we also have

$$
\left|1-\kappa x_{i} y_{j}\right| \geq a
$$

(15) is at most

$$
a^{-m^{2}} \frac{\Delta(x)^{2} \Delta(y)^{2}}{\left(1-\prod x_{i} y_{i}\right)^{2 n^{2}}} \prod_{i} \sqrt{\frac{1-x_{i}}{1-y_{i}} \frac{y_{i}}{x_{i}}}
$$


With $x_{i}=1-\xi_{i}, y_{i}=1-\eta_{i}$ and $r=\sum_{i}\left(\xi_{i}+\eta_{i}\right)$ again, we first integrate over $r<\delta$, where the small $\delta$ will be chosen below. Using (14) again, we see that the integrand is at most $A^{m}$ times

$$
a^{-m^{2}} \frac{\Delta(\xi)^{2} \Delta(\eta)^{2}}{\left(\sum_{i}\left(\xi_{i}+\eta_{i}\right)\right)^{2 n^{2}}} \prod_{i} \sqrt{\xi_{i} / \eta_{i}}
$$

(The factor $m^{2 n^{2}}$ coming from using (14) and a bound for $\prod \sqrt{y_{i} / x_{i}}$ appearing in (15) were absorbed into $A^{m}$.) When $\xi_{i}, \eta_{i}<1$ we have $\Delta(\xi)^{2}, \Delta(\eta)^{2}<1$, so integrating with respect to $r$ over $r<\delta$, using homogeneity, gives at most a constant times

$$
a^{-m^{2}} \int_{0}^{\delta} r^{2 m(m-1)-2 n^{2}+2 m-1} d r=a^{-m^{2}} \int_{0}^{\delta} r^{2 m^{2}-2 n^{2}-1} d r .
$$

(The integral of the last factor over $r=1$ equals $(\pi / 2)^{m} / \Gamma(2 m)$.) The integral is $O\left(\delta^{2 m^{2}}\right)$ since $m>n$, and so the above is exponentially small in $m$ if we choose $\delta^{2}<a$.

There remains the integral over the region $r>\delta$, and for this we use the representation (11). We are led to (15) with the second factor replaced by the absolute value of

$$
\left(\operatorname{det}\left(\frac{1}{1-\kappa x_{i} y_{j}}\right)\right)^{2} \text {. }
$$

From (14) we see that in this region the first factor in (15) is at most $(2 m / \delta)^{2 n^{2}}$. By (16) and the Hadamard inequality the square of the determinant has absolute value at most $a^{-2 m} m^{m}$. Therefore the integral over this region has absolute value at most

$$
\left(\frac{2 m}{\delta}\right)^{2 n^{2}} a^{-2 m} m^{m} \int_{0}^{1} \cdots \int_{0}^{1} \prod_{i} \sqrt{\frac{1-x_{i}}{1-y_{i}} \frac{y_{i}}{x_{i}}} \prod_{i} d x_{i} d y_{i} .
$$

The integral here is $A^{m}$, and so we have shown that the integral in the region $r>\delta$ is at most $A^{m} m^{m}$.

This is a bound for only one term we get when we differentiate $2 n^{2}-1$ times the integrand for $\mathcal{S}_{m}$. The number of factors in the integrand involving $\kappa$ is $O\left(\mathrm{~m}^{2}\right)$ so if we differentiate $2 n^{2}-1$ times we get a sum of $O\left(m^{4 n^{2}}\right)$ terms. In each of the other terms the denominator in the first factor has a power no larger than $2 n^{2}$ and at most $2 n^{2}$ extra factors appear which are of the form $\left(1-\kappa x_{i} y_{i}\right)^{-1},\left(1-\kappa x_{i}\right)^{-1}$, or $\left(1-\kappa y_{i}\right)^{-1}$. Each has absolute value at most $a^{-1}$, so their product is $O(1)$. It follows that we have the bound $A^{m} m^{m}$ for the sum of these integrals. Lemma 4 is established.

Proof of the Theorem. Suppose $\kappa \rightarrow \epsilon$ radially, where $\epsilon \neq 1$ is a root of unity. It is a primitive $n$th root of unity for some $n$. Then $\epsilon^{m} \neq 1$ when $m<n$ so Lemma 3 
applies for these $m$. Combining this with Lemma 4 and Lemma 2 gives

$$
\left(\frac{d}{d \kappa}\right)^{2 n^{2}-1} \mathcal{S} \approx \log (1-|\kappa|)^{-1} .
$$

Therefore $\mathcal{S}$ cannot be analytically continued beyond any such $\epsilon$, and these are dense in the unit circle.

Remark. From the proofs of Lemma 3 and 4 , with $2 n^{2}-1$ replaced by $\ell$ in both, one can see that $\mathcal{S}$ extends to a $C^{\ell}$ function of $\kappa$ up to the boundary except for the

$m$ th roots of unity with $m \leq \sqrt{(\ell+1) / 2}$. In particular, $\mathcal{S}$ extends to a function of class $C^{6}$ up to the boundary except for $\kappa=1$.

\section{Acknowledgments}

The authors wish to thank Bernie Nickel and Barry McCoy for their helpful comments. This work was supported by the National Science Foundation through grants DMS-1207995 (first author) and DMS-0854934 (second author).

\section{References}

[1] C. Andréief, Note sur une relation les intégrales définies des produits des fonctions, Mém. de la Soc. Sci., Bordeause 2 (1883), 1-14.

[2] M. Assis, S. Boukraa, S. Hassani, M. Van Hoeij, J.-M. Maillard, and B. M. McCoy, Diagonal Ising susceptibility: elliptic integrals, modular forms and CalabiYau equations. J. Phys. A: Math. Theor. 45 (2012), 075205 (32 pp).

[3] E. Basor and H. Widom, On a Toeplitz determinant identity of Borodin and Okounkov, Integral Equations and Operator Theory 37 (2000), 397-401.

[4] R. J. Baxter, Onsager and Kaufman's calculation of the spontaneous magnetization of the Ising model, J. Stat. Phys. 145 (2011), 518-548.

[5] A. Borodin and A. Okounkov, A Fredholm determinant formula for Toeplitz determinants, Integral Equations and Operator Theory 37 (2000), 386-396.

[6] A. Böttcher, One more proof of the Borodin-Okounkov formula for Toeplitz determinants, Integral Equations and Operator Theory 41 (2001), 123-125.

[7] S. Boukraa, S. Hassani, J.-M. Maillard, B. M. McCoy and N. Zenine, The diagonal Ising susceptibility, J. Phys. A: Math. Theor. 40 (2007), 8219-8236. 
[8] A. I. Bugrii, Correlation function of the two-dimensional Ising model on a finite lattice: I, Theor. and Math. Phys. 127 (2001), 528-548.

[9] A. I. Bugrii and O. O. Lisovyy, Correlation function of the two-dimensional Ising model on a finite lattice: II, Theor. and Math. Phys. 140 (2004), 987-1000.

[10] Y. Chan, A. J. Guttmann, B. G. Nickel and J. H. H. Perk, The Ising susceptibility scaling function, J. Stat. Phys. 145 (2011), 549-590.

[11] P. Deift, A. Its and I. Krasovsky, Toeplitz matrices and Toeplitz determinants under the impetus of the Ising model: Some history and some recent results, Commun. on Pure and Appl. Math. 66 (2013), 1360-1438.

[12] J. S. Geronimo and K. M. Case, Scattering theory and polynomials orthogonal on the unit circle, J. Math. Phys. 20 (1979), 299-310.

[13] A. J. Guttmann and I. G Enting, Solvability of some statistical mechanical systems, Phys. Rev. Letts. 76 (1996), 344-347.

[14] I. Lyberg and B. M. McCoy, Form factor expansion of the row and diagonal correlation functions of the two-dimensional Ising model, J. Phys. A: Math. Theor. 40 (2007), 3329-3346.

[15] B. M. McCoy and T. T. Wu, The Two-Dimensional Ising Model, Harvard University Press, 1973.

[16] B. M. McCoy, Advanced Statistical Mechanics, Oxford University Press, Great Clarendon Street, Oxford, 2010.

[17] B. M. McCoy, M. Assis, S. Boukraa, S. Hassani, J.-M. Maillard, W. P. Orrick and N. Zenine, The saga of the Ising susceptibility, in New Trends in Quantum Integrable Systems: Proceedings of the Infinite Analysis 09, eds. B. L. Feigin, M. Jimbo and M. Okado, World Scientific, 2010, pp. 287-306. [preprint available at arXiv:1003.0751.].

[18] B. Nickel, On the singularity structure of the 2D Ising model, J. Phys. A: Math. Gen. 32 (1999), 3889-3906.

[19] B. Nickel, Addendum to 'On the singularity structure of the 2D Ising model', J. Phys. A: Math. Gen. 33 (2000), 1693-1711.

[20] L. Onsager, Crystal statistics, I. A two-dimensional model with an order-disorder transition, Phys. Rev. 65 (1944), 117-149.

[21] L. Onsager, discussion, Nuovo Cimento 6 Suppl. (1949), 261. 
[22] W. P. Orrick, B. Nickel, A. J. Guttmann and J. H. H. Perk, The susceptibility of the square lattice Ising model: New developments, J. Stat. Phys. 102 (2001), 795-841.

[23] J. Palmer, Planar Ising Correlations, Progress in Mathematical Physics, Vol. 49, Birkhäuser, Boston, 2007.

[24] J. Stephenson, Ising-model spin correlations on the triangular lattice, J. Math. Phys. 5 (1964), 1009-1024.

[25] C. A. Tracy and H. Widom, Correlation functions, cluster functions, and spacing distributions for random matrices, J. Stat. Phys. 92 (1998), 809-835.

[26] N. S. Witte and P. J. Forrester, Fredholm determinant evaluations of the Ising model diagonal correlations and their $\lambda$ generalization, Studies in Appl. Math. 128 (2011), 183-223.

[27] T. T. Wu, B. M. McCoy, C. A. Tracy and E. Barouch, Spin-spin correlation functions for the two-dimensional Ising model: Exact theory in the scaling region, Phys. Rev. B13 (1976), 315-374.

[28] C. N. Yang, The spontaneous magnetization of the two-dimensional Ising model, Phys. Rev. 85 (1952), 808-816. 